\title{
A PILOT SURVEY FOR C III] EMISSION IN THE REIONIZATION ERA: GRAVITATIONALLY LENSED $z \sim 7-8$ GALAXIES IN THE FRONTIER FIELDS CLUSTER ABELL 2744
}

\author{
Adi Zitrin ${ }^{1,3}$, Richard S. Ellis ${ }^{1}$, Sirio Belli ${ }^{1}$, and Daniel P. Stark ${ }^{2}$ \\ ${ }^{1}$ Cahill Center for Astronomy and Astrophysics, California Institute of Technology, MC 249-17, Pasadena, CA 91125, USA; adizitrin@gmail.com \\ ${ }^{2}$ Steward Observatory, University of Arizona, 933 N Cherry Ave, Tucson, AZ 85721, USA \\ Received 2015 March 25; accepted 2015 April 28; published 2015 May 15
}

\begin{abstract}
We report results of a search for C III] $\lambda \lambda 1907,1909 \AA$ emission using Keck’s MOSFIRE spectrograph in a sample of $7 z_{\text {phot }} \sim 7-8$ candidates $(H \sim 27)$ lensed by the Hubble Frontier Field cluster Abell 2744. Earlier work has suggested the promise of using the $\mathrm{C}$ III] doublet for redshift confirmation of galaxies in the reionization era given that $\operatorname{Ly} \alpha(\lambda 1216 \AA)$ is likely attenuated by the neutral intergalactic medium. The primary challenge of this approach is the feasibility of locating $\mathrm{C}$ III] emission without advanced knowledge of the spectroscopic redshift. With an integration time of $5 \mathrm{hr}$ in the $H$ band, we reach a $5 \sigma$ median flux limit (in between the skylines) of $1.5 \times 10^{-18} \mathrm{ergs} \mathrm{cm}^{-2} \mathrm{~s}^{-1}$ but no convincing $\mathrm{C}$ III] emission was found. We also incorporate preliminary measurements from two other CLASH/HFF clusters in which, similarly, no line was detected, but these were observed to lesser depth. Using the known distribution of $\mathrm{OH}$ emission and the photometric redshift likelihood distribution of each lensed candidate, we present statistical upper limits on the mean total $\mathrm{C}$ III] rest-frame equivalent width (EW) for our $z \simeq 7-8$ sample. For a signal-to-noise ratio of 5 , we estimate that the typical $\mathrm{C}$ III] doublet rest-frame EW is, with $95 \%$ confidence, $<26 \pm 5 \AA$. Although consistent with the strength of earlier detections in brighter objects at $z \simeq 6-7$, our study illustrates the necessity of studying more luminous or strongly lensed examples prior to the launch of the James Webb Space Telescope.
\end{abstract}

Key words: cosmology: observations - galaxies: clusters: general - galaxies: evolution - galaxies: formation galaxies: high-redshift - gravitational lensing: strong

\section{INTRODUCTION}

The reionization of the intergalactic medium (IGM) represents a key phase in the evolution of the universe. Observations of high-redshift galaxies, which have charted a marked decline in the visibility of Ly $\alpha$ emission with redshift (Stark et al. 2010; Schenker et al. 2012), and those of $z>5$ quasars which trace the redshift-dependent Gunn-Peterson absorption (Fan et al. 2006), indicate that cosmic reionization was largely complete by $z \sim 6$. The duration of the reionization process is constrained by the polarization of the microwave background due to Thomson scattering by electrons in the ionized era; recent data from the Planck satellite and results derived from the abundance and luminosity distribution of the $z>6$ galaxy population now suggest that reionization was a rapid process which extended over $6<z<10$ (Planck Collaboration et al. 2015; Robertson et al. 2015).

While Ly $\alpha$ emission $(\lambda 1216 \AA)$ has proven to be the most valuable spectroscopic indicator for faint star-forming galaxies in the redshift range $4<z<6$ (e.g., Stark et al. 2010, 2011), resonant scattering by neutral gas in the IGM likely renders this line ineffective as a reliable probe beyond $z \simeq 6.5$. Despite much observational effort, there are currently very few convincing cases of detected Ly $\alpha$ emission beyond $z \simeq 7$ (Ono et al. 2012; Finkelstein et al. 2013; Schenker et al. 2014; Vanzella et al. 2014a; Oesch et al. 2015) and several distant star-forming galaxies reveal no emission despite heroic exposure times (Vanzella et al. 2014b). As a result, Stark et al. (2014b) proposed it may be feasible to use metallic lines in the ultraviolet (UV) as alternative spectroscopic indicators. Examining the spectra of 17 gravitationally lensed low-

\footnotetext{
${ }^{3}$ Hubble Fellow.
}

luminosity galaxies at $z \simeq 1.5-3$, they discuss the feasibility of searching for C III] $(\lambda \lambda 1907,1909 \AA)$ and C IV $(\lambda \lambda 1548$, $1550 \AA$ ) emission. Although such metallic lines are normally much weaker than Ly $\alpha$ in luminous systems, in young metalpoor low luminosity systems characteristic of those at high redshifts, these lines may become relatively more prominent. In their sample of $17 z \simeq 1.5-3$ galaxies, Stark et al. (2015) find $\mathrm{C}$ III] emission has an equivalent width (EW) which correlates with that of $\operatorname{Ly} \alpha$ and is typically 10 times weaker. As an encouraging proof of concept, Stark et al. (2014) recently claimed tentative detections of $\mathrm{C}$ III] emission in two $J \sim 25.2$ galaxies with pre-determined $\mathrm{Ly} \alpha$ emission at redshifts of $z=6.03$ and $z=7.21$.

However, as emphasized by Stark and collaborators, detecting $\left.\mathrm{C}_{\mathrm{III}}\right]$ emission in galaxies where its expected wavelength is a priori known from a $\mathrm{Ly} \alpha$ redshift is less challenging than searching for emission across a wider range of wavelength governed only by a photometric redshift likelihood distribution, and given the density of skylines. Motivated by the interest in exploring the potential of this, possibly the only, immediate route to spectroscopic progress in the reionization era, we have embarked on a statistical search. Our plan is examine the spectra of a sample of gravitationally lensed sources in the redshift range $z \sim 6.7-8.5$ derived from recent compilations in several massive clusters (e.g., Atek et al. 2014; Bradley et al. 2014; Coe et al. 2015; Zheng et al. 2014). Such a statistical approach is now possible due to the arrival of multislit near-infrared spectrographs such as Keck's Multi-Object Spectrometer For Infra-Red Exploration (MOSFIRE; McLean et al. 2012). In this paper, we examine the practicality of the method with realistic exposure times for the Frontier Field cluster Abell 2744, and also include preliminary data for two other CLASH/HFF clusters observed to shallower depths. 
The paper is organized as follows. In Section 2 we overview the sample, observations, and data reduction. In Section 3 we discuss the results, summarized in Section 4. Throughout the work we use a standard $\Lambda$ CDM cosmology with $\left(\Omega_{m 0}=0.3\right.$, $\Omega_{\Lambda 0}=0.7, H_{0}=100 h \mathrm{~km} \mathrm{~s}^{-1} \mathrm{Mpc}^{-1}$, with $\left.h=0.7\right)$, and magnitudes are given using the $\mathrm{AB}$ convention. Cluster names are abbreviated to "A" or "M" for Abell (e.g., Abell et al. 1989) and MAssive Cluster Survey (MACS; e.g., Ebeling et al. 2010) clusters, respectively, followed by their ID.

\section{DATA}

\subsection{Target Selection}

We constructed a sample of high- $z$ candidates magnified by the galaxy cluster A2744 using photometry from Hubble Frontier Fields program (Lotz et al. 2014). Candidates for inclusion in our multi-slit mask were derived from Zheng et al. (2014), Coe et al. (2015) and Atek et al. (2015, 2014, see also Ishigaki et al. 2015). We pre-selected targets of known magnification down to an apparent magnitude of $H_{160} \sim 28$ with photometric redshifts in the range $6.7<z<8.5$, corresponding to the visibility of C III] within MOSFIRE's $H$ band filter. We also included the $z \sim 9.8$ candidate from Zitrin et al. (2014) to explore the option of detecting the $\mathrm{C}$ IV $\lambda \lambda$ $(1548,1550) \AA$ doublet in the same band. This follows a promising detection of $\mathrm{C}$ IV emission at $z \simeq 7.05$ by Stark et al. (2015). Photometric redshift likelihood distributions, $P(z)$, were obtained with the Bayesian Photometric Redshift code (BPZ; Benítez et al. 2004; Coe et al. 2006; see also Zheng et al. 2014), covering the full redshift range available to $H S T$ (from $z=0.01$ to $z=12$ in $\Delta z=0.001$ increments). Due to slit-mask positioning constraints, only a subset of good candidates could be included on the MOSFIRE mask and priority was given to brighter galaxies. The final mask included eight high $z$ candidates as summarized in Table 1 . The $\left.\mathrm{C}_{\text {III }}\right]$ candidates lie mainly in the magnitude range $26.2<H<27.5$ and have photo- $z$ uncertainties of $\delta z \simeq 0.2-0.3$. The restframe UV luminosities corrected for lensing magnifications have a mean of $M_{\mathrm{UV}} \simeq-18.8$ and standard deviation of 1.4. As part of this campaign we also have begun observations of two further lensing clusters, MACS 0416 and A2261, drawing candidates from the catalogs of Bradley et al. (2014) and Coe et al. (2015, see also McLeod et al. 2014). As the photometric redshift distributions of these galaxies are somewhat less secure (especially for A2261), and since our observations of these clusters are significantly shallower, they currently provide less useful constraints on the presence of $\mathrm{C}$ III], although we incorporate the results in this paper. We also list these additional sources in Table 1. Figure 1 summarizes the redshift and UV luminosity distribution of the total sample in the context of the $H$-band window available for detecting $\mathrm{C}$ III] with MOSFIRE.

\subsection{Observations and Data Reduction}

Observations with MOSFIRE on the Keck I telescope were undertaken on 2014 September 16, November 25, and November 27. A total exposure of $5 \mathrm{hr}$ was obtained for A2744. Thus far only 2.7 and $2.2 \mathrm{hr}$ have been secured for A2261 and M0416, respectively. Median seeing varied between $\sim 0.5$ and 0." 8 . Each exposure comprised $120 \mathrm{~s}$ integrations with an $\mathrm{AB}$ dithering pattern of \pm 1 ". 25 along the slit. On each mask, one slit was assigned to an alignment star, in order to track possible positional drifts and transparency changes.

Data reduction was performed using the standard MOSFIRE reduction pipeline. ${ }^{4}$ For each flat-fielded slit we extracted the 1D spectrum using a 11 pixel boxcar centered on the expected position of the target. A similar procedure was adopted in quadrature to derive the $1 \sigma$ error distribution. The addition of data from different nights was performed by inverse-variance averaging the calibrated 1D spectra. To obtain the $1 \sigma$ flux limits for $\mathrm{C}$ III], we assumed a marginally resolved line width (Stark et al. 2015) corresponding to three MOSFIRE pixels $(\sim 5 \AA$ in the $H$ band) and summing in quadrature the $1 \mathrm{D} \sigma$ spectrum within the three-pixel window.

Spectrophotometric standard stars were observed twice a night in similarly good conditions. We scaled a Vega model ${ }^{5}$ to each standard star to determine a wavelength-dependent flux calibration using the procedure described in Vacca et al. (2003), which more accurately traces telluric corrections free from stellar absorption features. Our various flux calibrations on each night agree to within $15 \%$ and are also consistent with the nominal MOSFIRE calibration files (C. Steidel 2015, private communication) to within $\sim 10 \%$. The calibrated spectra of alignment stars (incorporated on our multi-slit mask) from different nights also agree to within $1 \%-8 \%$, and are typically within $\sim 20 \%$ of the flux level expected from their $H_{160}$ photometry, after aperture corrections for slit losses. Using our adopted calibration, the median $5 \sigma$ detection limit achieved in between the $\mathrm{OH}$ skylines for our A2744 exposure is $1.5 \times 10^{-18} \mathrm{cgs}$. This line flux limit is comparable with that achieved by Stark et al. (2015) using MOSFIRE. In their $3.1 \mathrm{hr}$ exposure, they report a limiting flux $(5 \sigma)$ of $1.8 \times 10^{-18} \mathrm{cgs}$.

\section{RESULTS}

All reduced spectra were visually inspected given the expected wavelength range where $\mathrm{C}_{\mathrm{III}}$ ] might be visible according to the photometric redshift likelihood function. No convincing line was seen for any of the seven $\mathrm{C}$ III] candidates. We thus seek to determine the likely range of fluxes and EW for $\mathrm{C}_{\mathrm{III}}$ ] consistent with our non-detections. ${ }^{6}$ In other words, we estimate the probability of detecting at least one $\left.\mathrm{C}_{\mathrm{III}}\right]$ line in our survey, as a function of a given mean total flux and EW. Since C III] is a doublet, we assume a line ratio C III] 1907/ $1909 \AA$ of 1.4 (Stark et al. 2015). We consider total line strengths in the range $0-4 \times 10^{-18} \mathrm{ergs} \mathrm{cm}^{-2} \mathrm{~s}^{-1}$ in $5 \times 10^{-20} \mathrm{ergs} \mathrm{cm}^{-2} \mathrm{~s}^{-1}$ increments as illustrated in Figure 2. For each doublet line, and for each redshift step, we checked if its input flux would exceed a certain detection significance $(x \sigma)$ in the corresponding wavelength in the observer frame, where $x$ is a chosen signal-to-noise ratio. For a fixed line flux, the likelihood of having a detection with $x \sigma_{k}$ significance in the examined slit $k$ is given by the number of spectral pixels with positive detections over the total number of pixels, weighted by the redshift probability function $P(z)$. The relevant expression

\footnotetext{
4 http://www2.keck.hawaii.edu/inst/mosfire/drp.html

5 http://kurucz.harvard.edu/stars/vega/

6 We note that A2744 was also observed with the same mask for $2 \mathrm{hr}$ in the $Y$ band, searching for $\operatorname{Ly} \alpha$. No line was detected and these data will be presented elsewhere.
} 
Table 1

The Sample

\begin{tabular}{|c|c|c|c|c|c|c|c|}
\hline ID & $\alpha$ (degree) & $\delta$ (degree $)$ & Phot- $z$ & $H_{160}$ & $\mu$ & $\beta$ & $M_{\mathrm{UV}, 1500}$ \\
\hline A2744-YD7 $7^{\mathrm{a}, \mathrm{b}}$ & 3.603397 & -30.382256 & $8.3_{-0.1}^{+0.1}$ & $26.17 \pm 0.03$ & $1.4_{-0.1}^{+0.7}$ & $-1.38 \pm 1.86$ & $-20.65_{-0.08}^{+0.54}$ \\
\hline A2744-ZD $3^{\mathrm{a}, \mathrm{b}, \mathrm{c}}$ & 3.606477 & -30.380993 & $7.7_{-0.3}^{+0.2}$ & $26.45 \pm 0.04$ & $1.3_{-0.1}^{+1.0}$ & $-1.14 \pm 0.26$ & $-20.19_{-0.19}^{+0.85}$ \\
\hline A2744-ZD9 ${ }^{\mathrm{a}}$ & 3.603208 & -30.410368 & $7.0_{-0.2}^{+0.2}$ & $26.48 \pm 0.04$ & $3.4_{-0.8}^{+0.8}$ & $-1.17 \pm 0.23$ & $-18.88_{-0.37}^{+0.37}$ \\
\hline A2744-ZD7A2 ${ }^{\mathrm{a}}$ & 3.592160 & -30.409925 & $7.3_{-0.5}^{+0.2}$ & $28.18 \pm 0.04$ & $6.4_{-2.2}^{+7.8}$ & $-1.29 \pm 1.22$ & $-16.63_{-0.42}^{+1.34}$ \\
\hline A2744-YD8 ${ }^{a, b, c}$ & 3.596096 & -30.385832 & $8.1_{-0.1}^{+0.2}$ & $26.65 \pm 0.04$ & $1.9_{-0.2}^{+1.0}$ & $-1.84 \pm 1.64$ & $-19.86_{-0.13}^{+0.57}$ \\
\hline Atek-3772 & 3.5978343 & -30.395960 & $7.0_{-0.6}^{+0.3}$ & $27.45 \pm 0.05$ & $\sim 6.8$ & $-1.77 \pm 1.00$ & $-17.51_{-0.24}^{+0.24}$ \\
\hline Atek-5918,e & 3.5951375 & -30.381131 & $7.7_{-0.6}^{+0.6}$ & $26.92 \pm 0.02$ & $\sim 3.5$ & $-1.07 \pm 0.19$ & $-18.65_{-0.27}^{+0.27}$ \\
\hline A2744-JDB ${ }^{\mathrm{d}}$ & 3.5950200 & -30.400750 & $9.8_{-0.4}^{+0.2}$ & $27.30 \pm 0.07$ & $11.3_{-2.5}^{+4.8}$ & $\ldots$ & $\sim-17.6$ \\
\hline A2261-0450 ${ }^{\mathrm{f}}$ & 260.6124593 & 32.1438429 & $6.8_{-0.3}^{+0.2}$ & $25.5 \pm 0.06$ & $\sim 5.6$ & $-1.85 \pm 0.15$ & $-19.50_{-0.23}^{+0.23}$ \\
\hline A2261-0731 ${ }^{\mathrm{f}}$ & 260.6232556 & 32.1393984 & $6.9_{-5.9}^{+1.0}$ & $27.9 \pm 0.22$ & $\sim 7.7$ & $-1.00 \pm 0.67$ & $-16.65_{-0.34}^{+0.34}$ \\
\hline A2261-0772 ${ }^{\mathrm{f}}$ & 260.6059024 & 32.1388049 & $6.5_{-5.4}^{+0.8}$ & $27.4 \pm 0.19$ & $\sim 6.3$ & $-2.17 \pm 0.64$ & $-17.35_{-0.30}^{+0.30}$ \\
\hline A2261- $0187^{\mathrm{f}}$ & 260.6073833 & 32.1495175 & $7.5_{-1.2}^{+0.4}$ & $27.0 \pm 0.13$ & $\sim 2.9$ & $-1.18 \pm 1.47$ & $-18.89_{-0.25}^{+0.25}$ \\
\hline MACS $0416-0036^{\mathrm{f}}$ & 64.0260447 & -24.0509958 & $7.0_{-6.0}^{+1.2}$ & $26.8 \pm 0.16$ & $\sim 1.3$ & $-0.56 \pm 0.45$ & $-19.46_{-0.45}^{+0.45}$ \\
\hline Zheng- $4008^{g}$ & 64.0603330 & -24.064960 & $7.7_{-0.3}^{+0.3}$ & $27.85 \pm 0.08$ & $2.2_{-0.3}^{+0.3}$ & $-3.49 \pm 1.33$ & $-18.59_{-0.28}^{+0.28}$ \\
\hline FFC2-1151-4540 & 64.0479780 & -24.081678 & $8.3_{-0.2}^{+0.2}$ & $26.59 \pm 0.03$ & $1.8_{-0.5}^{+0.5}$ & $-1.44 \pm 0.69$ & $-19.93_{-0.31}^{+0.31}$ \\
\hline
\end{tabular}

Note. Column 1: dropout's ID and references. The first work cited for each object represents the original source of photometric data and analysis, although in some cases we made adjustments to enhance consistency across the sample. Columns 2 and 3: R.A. and decl. in J2000.0. Column 4: photometric redshift and 95\% errors. Column 5: HST's apparent $H_{160}$-band magnitude. Column 6: lensing magnification. If no error is listed a nominal $\sim 20 \%$ error is adopted (Zitrin et al. 2015). Column 7: UV-slope, $\beta$ ( $1 \sigma$ errors), calculated by a weighted least-squares fit. Column 8: absolute magnitude, $M_{\mathrm{UV}}$, at $\lambda=1500 \AA$, calculated from the said $F_{\lambda} \propto \lambda^{\beta}$ fit, where the error includes in quadrature the discrepancy from the absolute magnitude obtained by translating the flux in the WFC 3 band containing the redshifted $\lambda=1500 \AA$, and the propagated photometric and magnification errors.

${ }^{a}$ Zheng et al. (2014).

${ }^{\mathrm{b}}$ Coe et al. (2015).

${ }^{c}$ Atek et al. $(2015,2014)$.

${ }^{\mathrm{d}}$ Zitrin et al. (2014). C IV target.

${ }^{\mathrm{e}}$ Our independent photo- $z$ estimate permits a solution at $z \simeq 2$.

${ }^{\mathrm{f}}$ Bradley et al. (2014).

${ }^{\mathrm{g}} \mathrm{W}$. Zheng (private communication; see also Infante et al., in preparation).

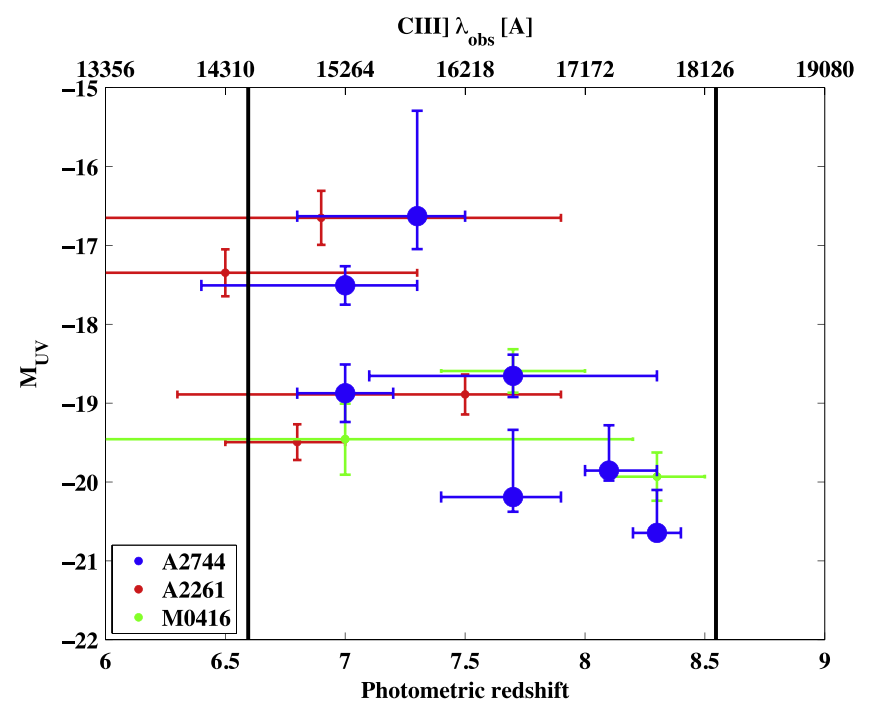

Figure 1. Distribution of our candidate lensed galaxies in photometric redshift and UV absolute magnitude for A2744 and the two other clusters. Black lines denote the window within which $\mathrm{C}$ III] would be visible at a wavelength indicated on the top axis.

for slit $k$ can be formulated as:

$$
P_{\mathrm{det}, k}\left(F_{\mathrm{in}}, x\right)=\frac{\sum_{i} P_{k}\left(z_{i}\right) \Theta\left(F_{\mathrm{in}}, x, \sigma_{k}, z_{i}\right)}{\sum_{i} P_{k}\left(z_{i}\right)},
$$

where the sum is over all the redshift steps $z_{i}(z=0$ to $z=12$ in 0.001 increments), and $\Theta$ is defined as

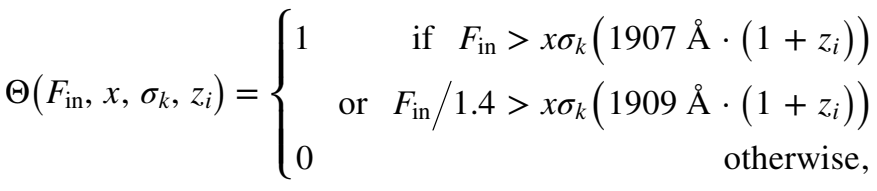

and we define $\sigma_{k}=\infty$ for $z_{i}$ steps placing the line outside the MOSFIRE $H$ band. $P_{\text {det, } k}$ therefore provides a conditional probability, i.e., the chance of detecting at least one of the two $\mathrm{C}_{\text {III] }}$ lines for a given target $k$ with redshift probability distribution $P_{k}(z)$, given the limiting noise in our spectra for the mask, $\sigma_{k}(\lambda)$ (see Section 2), and as a function of the input line flux $F_{\text {in }}$ and the detection significance $x$. Finally, the probability of detecting at least one line of a given flux $F_{\text {in }}$ over the entire sample is:

$$
P_{\text {sample }}\left(F_{\text {in }}, x\right)=1-\prod_{k}\left(1-P_{\text {det }, k}\left(F_{\text {in }}, x\right)\right),
$$

where the product is over all slits.

We repeat the above process also in terms of restframe EW, where in each iteration, instead of running over a range of input fluxes, we run over a range of restframe EWs, translated in each iteration, for each object individually, to the corresponding input flux.

Figure 3 shows the probability, for both 3 and $5 \sigma$ detections, of finding at least one line in our A2744 survey as a function of the mean total $\mathrm{C}$ III] flux and rest-frame EW. For example we 

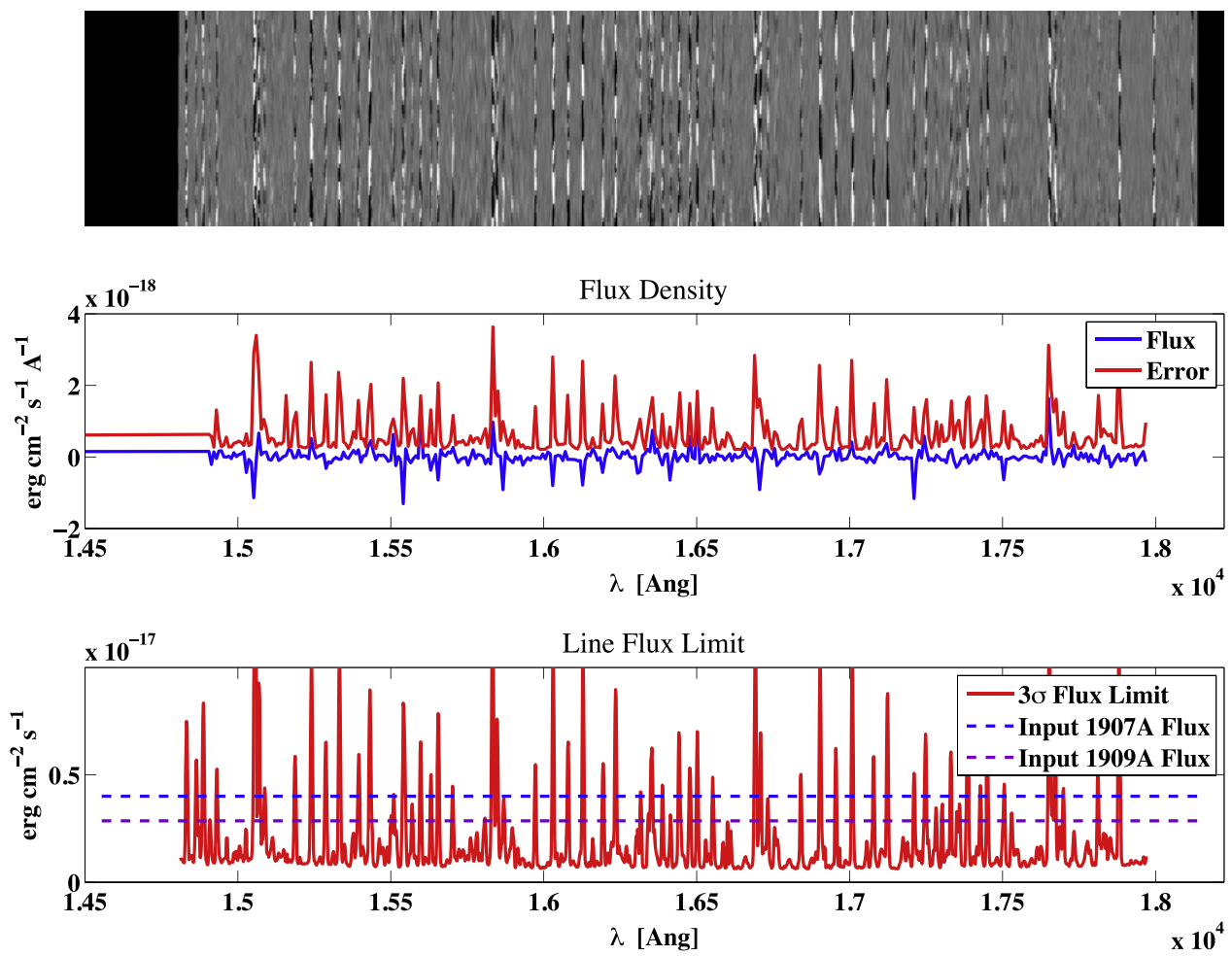

Figure 2. Illustration of the data. The top panel shows an arbitrary reduced slit in A2744, centered vertically on the C III] candidate. The middle panel shows the extracted 1D spectrum (blue), and its $1 \sigma$ error (red) — both smoothed here for illustrative purposes. The bottom panel shows an example of a step in the procedure for determining the upper-limit typical $\mathrm{C} \mathrm{III]}$ line flux in our sample. In red we show the $3 \sigma$ line flux limit, and the dashed blue and purple horizontal lines show fiducial input $\mathrm{C}$ III] line fluxes. For each such iteration we measure the fraction (in wavelength) in which the input flux is higher than the observational limit, weighted by the photometric redshift distribution. This indicates the chance of seeing $\mathrm{C}$ III], as elaborated in Section 3.

have a $95 \%$ chance of detecting at least one $\mathrm{C}$ III] line in our MOSFIRE survey at $5 \sigma$ significance, if the typical $\left.\mathrm{C}_{\mathrm{III}}\right]$ $\lambda 1907 \AA$ line flux is $\simeq 1.5 \times 10^{-18} \mathrm{ergs} \mathrm{cm}^{-2} \mathrm{~s}^{-1}$ (total doublet flux of $\simeq 2.6 \times 10^{-18} \mathrm{ergs} \mathrm{cm}^{-2} \mathrm{~s}^{-1}$ ), or equivalently, if the restframe EW for the combined C III] doublet is $26 \pm 5 \AA$ or higher. In this estimate we have included limits from the shallower exposures on A2261 and M0416, but the results remain similar (to within typically 5\%) if the sample is restricted to A2744for which photometric redshift errors are typically smaller and the observations are significantly deeper. Errors were propagated assuming our adopted $20 \%$ uncertainty in the flux calibration.

For comparison, Stark et al. (2015) detected, with $3.3 \sigma$, a $\lambda 1909 \AA \mathrm{C}$ III] line of $\simeq 4.2 \pm 1.2 \times 10^{-18} \mathrm{ergs} \mathrm{cm}^{-2} \mathrm{~s}^{-1}$ (total estimated $\mathrm{C}_{\text {III] }}$ flux $\simeq 1.1 \pm 0.3 \times 10^{-17}$ ) in a $z=6.03$ galaxy $(J=25.2)$, and a $2.8 \sigma \mathrm{C}$ III] detection of likely $\lambda 1909 \AA$ of $\simeq 0.9 \pm 0.3 \times 10^{-18} \mathrm{ergs} \mathrm{cm}^{-2} \mathrm{~s}^{-1} \quad$ (total estimated $\mathrm{C}$ III] flux $\left.\simeq 2.3 \pm 0.5 \times 10^{-18}\right)$ in a $z=7.21$ galaxy $(J=25.2)$. The total $\mathrm{C}$ III] restframe EWs of these detections are $22.5 \pm 7.1 \AA$ and $7.6 \pm 2.8 \AA$, respectively.

While our observational limits are deep enough to recover similar line fluxes to those found by Stark et al. (2015, see also Stark et al. 2014), our galaxies are fainter ( 27 AB), so that our limits on the rest-frame EWs are less constraining. Assuming a $\left.\mathrm{C}_{\mathrm{III}}\right] \mathrm{EW}$ of 22.5(7.6) $\AA$ as was found by Stark, we have $\sim 99.9 \%(10 \%)$ chance of detecting at least one such line in our sample with $3 \sigma$, or $\sim 90 \%(0 \%)$ for $5 \sigma$. Thus, it is quite likely that the primary reason for the non-detection in our survey is that, on average, the present sample is significantly fainter than those targeted by Stark et al., which also had the benefit of secure Ly $\alpha$-based redshifts. The main conclusion of our limits seen in Figures 3 and 4 is that even with a more ambitious spectroscopic campaign that would likely increase the exposure time by a factor $\times 4$ (corresponding to a three night integration on one mask), only more luminous $z \simeq 7-8$ galaxies in the reionization era would appear to be amenable for study with any reliability. Alternatively, brighter and/or more highly magnified examples, such as those close to the critical line of a foreground cluster, might provide promising targets although generally such sources are rare. It is interesting to note the non-detection (and upper limit) on $\mathrm{C}$ III] emission recently claimed by Watson et al. (2015) for a brighter source with $H=24.7$, magnified by $\mu \sim 10$ at $z=7.5$, showing that even for significantly brighter objects $\mathrm{C}$ III] detection can be challenging. Searching for bright magnified dropouts in a very large sample of clusters, for example, is desirable for progress with current facilities and might help deliver James Webb Space Telescope with first light targets.

For completeness, we also calculate the limit on the C IV $\lambda \lambda(1548,1550) \AA$ doublet for the $z \simeq 9.8$ multiply imaged object discovered by Zitrin et al. (2014) behind A2744 (Figure 3). Stark et al. (2014) found prominent C IV emission in some of the $z \sim 2$ galaxies they targeted, and highlighted $\mathrm{C}$ IV as an additional promising diagnostic for high-redshift galaxies. Typically they found C IV line fluxes only a factor of about 2 weaker than those of $\mathrm{C}_{\mathrm{III}}$. At the proposed redshift of the Zitrin et al. (2014) object, the doublet would be readily resolved and we assume both lines have equal strength. In this case, we determine that, with $\sim 90 \%$ confidence, the line flux for either one of the two C IV lines for a detection significance of $5 \sigma$ is less than 

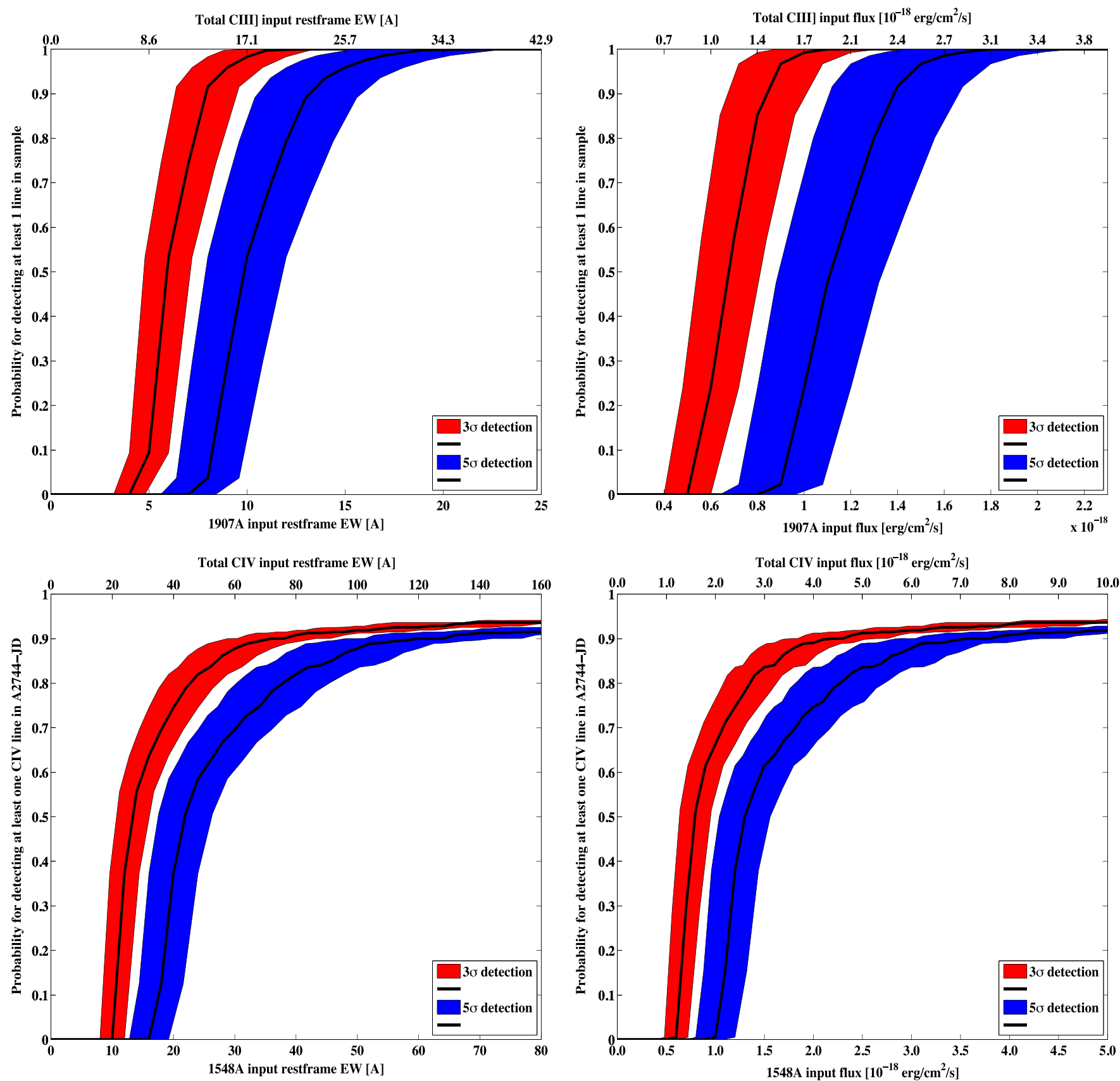

Figure 3. Top left: probability to detect at least one $\mathrm{C}$ III] line in our sample, as a function of the total (top axis) and $1907 \AA$ (bottom axis) C $\mathrm{mII}]$ rest-frame equivalent width. We plot the probabilities for detection thresholds of 3, and $5 \sigma$, and the shaded regions indicate the errors based on our adopted $20 \%$ flux calibration error. Top right: a similar plot in terms of the total and $1907 \AA ̊$ line flux. Bottom: probability to detect at least one of the two C iv lines in the $z \sim 9.8$ object (Zitrin et al. 2014$)$, as a function of line flux and restframe EW.

$\simeq 3.6 \times 10^{-18} \mathrm{ergs} \mathrm{cm}^{-2} \mathrm{~s}^{-1}$, and the rest-frame EW less than $\simeq 32 \AA$. This translates to a magnification-corrected, total $\mathrm{C}$ IV luminosity of $\lesssim 3.9 \times 10^{41} \mathrm{erg} \mathrm{s}^{-1}$ at $z=9.8$. It would be interesting to investigate further the properties of $\mathrm{C}$ IV emission in a larger sample. Note also Stark et al. (2015) have now detected a promising a C IV $\lambda 1548 \AA$ line in a $z \simeq 7.05$ object, corresponding to a restframe $\mathrm{EW}$ of $\simeq 18.1 \AA$ (a total $\mathrm{C}_{\mathrm{IV}}$ restframe EW of $38 \AA$ ). As in the $\left.\mathrm{C}_{\mathrm{III}}\right]$ case, despite reaching deep enough to detect a similar line flux, $\simeq 4.1 \times 10^{-18} \mathrm{ergs} \mathrm{cm}^{-2} \mathrm{~s}^{-1}$, in terms of EW the non-detection is consistent with the limits obtained from the single $z \sim 10$ object.

\section{CONCLUSIONS}

Given the attenuation of $\operatorname{Ly} \alpha$ by neutral gas in the reionization era, the $\mathrm{C}$ III] doublet has been proposed as a promising route toward spectroscopic verification and study of high-redshift candidates (Stark et al. 2015, 2014). We report results from a short campaign with Keck/MOSFIRE to assess the prospects of detecting $\mathrm{C}$ III] lines in a sample of faint gravitationally lensed $z \sim 7-8$ galaxies where $\operatorname{Ly} \alpha$ is not seen and thus the search window in wavelength is much larger than in earlier work. We observed 14 high- $z$ candidates magnified by three galaxy clusters. For our deepest field (A2744, with seven $\mathrm{C}_{\text {IIII }}$ candidates), we reached a $5 \sigma(3 \sigma)$ flux limit of 


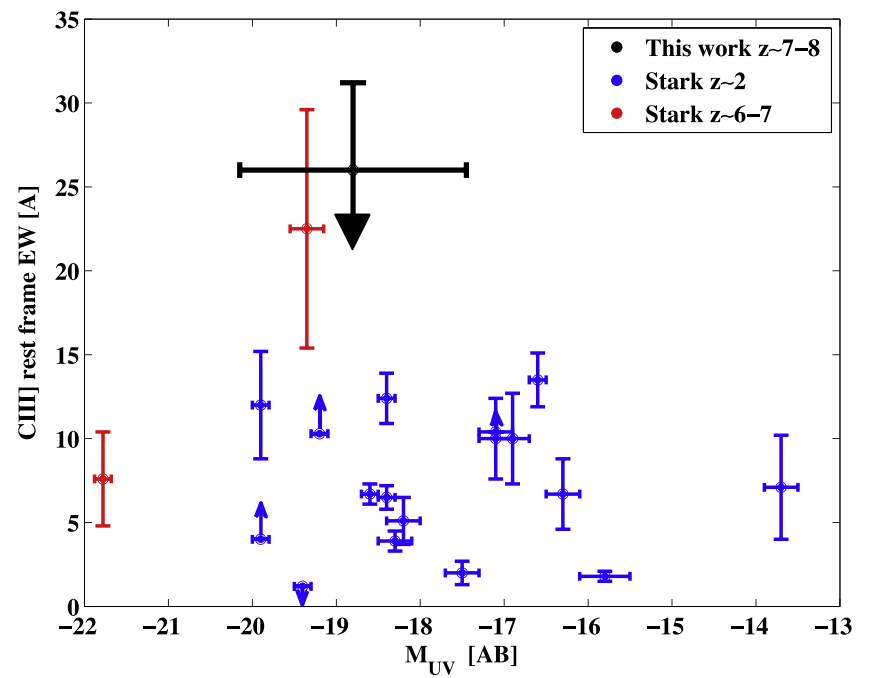

Figure 4. Rest-frame EW as a function of absolute UV magnitude, of previous measurements of C III] from Stark et al. $(2015,2014)$. The black error bar and arrow shows the $(95 \%$ C.L.) limit obtained in this work.

$1.5(0.9) \times 10^{-18} \mathrm{ergs} \mathrm{cm}^{-2} \mathrm{~s}^{-1}$ but did not detect any convincing line. Using a statistical method employing data from our collective campaign, we provide upper limits on the typical $\mathrm{C}$ III] line flux and its rest-frame EW. Although our limits reach the line fluxes observed in some actual $\mathrm{C}_{\text {III }}$ ] detections claimed in the recent literature, because our sample is significantly fainter in apparent magnitude, we only marginally reach the expected EWs based on these recent detections. This demonstrates the challenge of continuing the present investigation with current observing facilities unless either (i) brighter or more strongly-lensed sources are targeted and/or (ii) the C III] is found to be more prominent in intrinsically fainter systems (e.g., Stark et al. 2014b). More data is needed to test this latter suggestion.

We thank the reviewer of this work for important comments. A.Z. is grateful for data products generated by Dan Coe and the CLASH team (PI: M. Postman), and for data supplied to us by Wei Zheng, Alberto Molino and group (HST grant AR 13279; PI: W. Zheng). A.Z. thanks Carrie Bridge and Adam Miller for helpful discussions, and Chuck Steidel for sharing his nominal calibration files. Support for this work was provided by NASA through Hubble Fellowship grant \#HST-HF2-51334.001 A awarded by STScI, which is operated by the Association of
Universities for Research in Astronomy, Inc. under NASA contract NAS 5-26555. This work is in part based on previous observations made with the NASA/ESA Hubble Space Telescope. The data presented herein were obtained at the $\mathrm{W}$. M. Keck Observatory. The authors wish to recognize and acknowledge the very significant cultural role and reverence that the summit of Mauna Kea has always had within the indigenous Hawaiian community. We are most fortunate to have the opportunity to conduct observations from this mountain.

\section{REFERENCES}

Abell, G. O., Corwin, H. G., Jr., \& Olowin, R. P. 1989, ApJS, 70, 1

Atek, H., Richard, J., Kneib, J.-P., et al. 2014, ApJ, 786, 60

Atek, H., Richard, J., Kneib, J.-P., et al. 2015, ApJ, 800, 18

Benítez, N., Ford, H., Bouwens, R., et al. 2004, ApJS, 150, 1 Bradley, L. D., Zitrin, A., Coe, D., et al. 2014, ApJ, 792, 76 Coe, D., Benítez, N., Sánchez, S. F., et al. 2006, AJ, 132, 926 Coe, D., Bradley, L., \& Zitrin, A. 2015, ApJ, 800, 84 Ebeling, H., Edge, A. C., Mantz, A., et al. 2010, MNRAS, 407, 83

Fan, X., Strauss, M. A., Becker, R. H., et al. 2006, AJ, 132, 117

Finkelstein, S. L., Papovich, C., Dickinson, M., et al. 2013, Natur, 502, 524 Ishigaki, M., Kawamata, R., Ouchi, M., et al. 2015, 799, 12

Lotz, J., Mountain, M., Grogin, N. A., et al. 2014, in American Astronomical Society Meeting Abstracts, 223, 254.01

McLean, I. S., Steidel, C. C., Epps, H. W., et al. 2012, in Proc. SPIE, 8446, $84460 \mathrm{~J}$

McLeod, D. J., McLure, R. J., Dunlop, J. S., et al. 2014, arXiv:1412.1472

Oesch, P. A., van Dokkum, P. G., Illingworth, G. D., et al. 2015, arXiv:1502.05399

Ono, Y., Ouchi, M., Mobasher, B., et al. 2012, ApJ, 744, 83

Planck Collaboration, Ade, P. A. R., Aghanim, N., et al. 2015, arXiv:1502.01589

Robertson, B. E., Ellis, R. S., Furlanetto, S. R., \& Dunlop, J. S. 2015, ApJL, 802, L19

Schenker, M. A., Ellis, R. S., Konidaris, N. P., \& Stark, D. P. 2014, ApJ, 795, 20

Schenker, M. A., Stark, D. P., Ellis, R. S., et al. 2012, ApJ, 744, 179

Stark, D. P., Ellis, R. S., Chiu, K., Ouchi, M., \& Bunker, A. 2010, MNRAS, 408, 1628

Stark, D. P., Ellis, R. S., \& Ouchi, M. 2011, ApJL, 728, L2

Stark, D. P., Richard, J., Charlot, S., et al. 2015, MNRAS, 450, 1846

Stark, D. P., Richard, J., Siana, B., et al. 2014, MNRAS, 445, 3200

Stark, D. P., Walth, G., Charlot, S., et al. 2015, arXiv:1504.06881

Vacca, W. D., Cushing, M. C., \& Rayner, J. T. 2003, PASP, 115, 389

Vanzella, E., Fontana, A., Pentericci, L., et al. 2014b, Msngr, 157, 46

Vanzella, E., Fontana, A., Zitrin, A., et al. 2014a, ApJL, 783, L12

Watson, D., Christensen, L., Kraiberg Knudsen, K., et al. 2015, Natur, 519,327

Zheng, W., Shu, X., Moustakas, J., et al. 2014, ApJ, 795, 93

Zitrin, A., Fabris, A., Merten, J., et al. 2015, ApJ, 801, 44

Zitrin, A., Zheng, W., Broadhurst, T., et al. 2014, ApJL, 793, L12 